\title{
An Overview of Optical Control Plane and Novel Unified Control Plane Architecture for IP/WDM Networks
}

\author{
Abdullah Alsulami \\ Monash University, Australia \\ abu7ala1@hotmail.com
}

Abstract: Considering that this is an evolving subject area with continuously proposals and recommendations being developed by some organizations such as International Telecommunication Union (ITU), the Optical Interworking Forum (OIF), and the Internet Engineering Task Force (IETF).

This research paper discusses and provides an overview of the Optical Control Plane and Novel Unified Control Plane for IP over WDM architecture and the current protocols that are used in control plane in optical networks as well as delves the research into a new Novel Unified Control Plane for IP over WDM architecture networks.

\section{INTRODUCTION}

The involvement of the optical communications in the past two decennary to provide the resilience against potential fiber or node failures, to provide intelligence required for efficient point-and-click provisioning, and to provide transmission capacities to higher transport levels. Due to the emergence of optical network elements, which makes it possible, that carries the intelligence required to efficiently managing such networks. The deployments in the recent years of Wavelength Division Multiplexed (WDM) which is based on the optical transport networks have met the defy of accommodating the phenomenal extension of the IP data traffic while providing novel services that are the restoration and rapid provisioning of very high bandwidth on demand and bandwidth circuits (Ellinas et al. 2011).

One of the most critical part in the Optical Control Plane is the consideration of designing and implementing the transport network in an effective control plane that can be utilized to build a manageable, scalable, and dynamic backbone network to support reliable services and cost-efficient to its customers. Nowadays, we can say that some functionality such as point-and-click provisioning for a connection, restoration, and protection against faults and traffic engineering are the Optical Control Plane responsibilities. These are functionalities that reduce operational costs, allow the introduction of novel application and services, and optimize network performance as well as multi-vendor interoperability across various carrier networks (Ellinas et al. 2011).

This research paper discusses and provides an overview of the Optical Control Plane and Novel Unified Control Plane for IP over WDM architecture and the current protocols that are used in control plane in optical networks as well as delves the research into a new Novel Unified Control Plane for IP over WDM architecture networks.

The existing link management, signaling, and routing protocols are also presented in an overview of the GMPLS framework, which obligates the functionalities of control plane networks.

\section{OVERVIEW OF THE OPTICAL CONTROL PLANE}

Distributed management schemes for the IP networks such as Multi-Protocol Label Switching MPLS are used to supply the necessary of the control plane to ensure manage the network resources, maintain connections, and automated provisioning as well as including the providing of Traffic Engineering (TE) and Quality of Service (QoS) (Ellinas et al. 2011). In the past a few years, some industry organizations such as IETF and OIF have been working on extending the framework of the MPLS to support devices that perform switching 
and packet switching in time, Generalized Multi-Protocol Label Switching (GMPLS), and wavelength. GMPLS is the extensions of routing protocols and signaling that are developed for supports the new feature of link management and MPLS Traffic engineering (Awduche \& Rekhter 2001; Ellinas et al. 2011). So, now GMPLS can be applied for the wavelength-routed optical networks as a control plane.

\section{CURRent Protocols USED For OCP NETWORKS}

In this section the current protocols are used in the Optical Control Plane Networks are listed and discussed.

\section{Link Management Protocol}

In GMPLS, the Link Management Protocol (LMP) is primarily holding the responsibility for neighbor discovery which means that the automated determination of the connectivity in the network. Thus, it is used for resource discovery, transparent network architectures, and up-to-date topology in the network (Lang 2005). The LMP also performs some other tasks including link fault isolation, management of the control channel, and link bundling. Such information of link identification parameters, physical properties of a fiber link interconnecting the nodes that are collected by neighbor discovery protocol is exchanged regarding these parameters to be achieved via in-band signaling, via a completely separate out-of-band data communications network (DCN), or via out-of-band control channel. After discovering the network topology, the information is either distributed to the network nodes using distributed network control DNC to be applied to determine the routing paths for various connections by the routing protocol, or the information will be kept at the central location (Central Network Manager CNM) (Ellinas et al. 2011).

The Link Management Protocol uses Hello and Configuration messages as periodic exchange between neighbor nodes which gives each nodes the required routing information about its neighbors, and allow it to monitor the health of the communication channel (Kompella, Berger \& Rekhter 2005). The link bundling technique is used to minimize the information exchange between nodes if they are connected with large number of fiber optic links (Kompella, Berger \& Rekhter 2005).

\section{Generalized Multi-Protocol Label Switching (Gmpls) Routing Protocol}

The Generalized Multi-Protocol Label Switching GMPLS routing protocol uses the Open Shortest Path First with Traffic Engineering (OSPF-TE) which is the extension of the MPLS routing protocol (Kompella, Katz \& Yeung 2003). The distributed OSPF is a link-state uses Dijkstra's algorithm to identify the shortest routing path from the routing table between the nodes (Dijkstra 1959; Ellinas et al. 2011). Each routing table contains the link parameters such as the link costs, and a complete network topology that is created by exchanging the information using the Link State Advertisements (LSAs) (Kompella, Katz \& Yeung 2003), which is required in order to successfully route an optical connections (Strand 2005).

\section{Generalized Multi-Protocol Label Switching (GMPLS) Signaling Protocol}

In the GMPLS Signaling, there are two types of the signaling protocols are used in the optical networks that are signaling between intermediate network nodes which take place in the Network-Network Interface (NNI), and signaling between the transport network and client which take place at the User-Network Interface (UNI) (Ellinas et al. 2011; Swallow et al. 2005). At the User-Network Interface (UNI) the signaling is used by the clients in order to request the connections across the transport network specifying some parameters such as the protection type for the connection, the Class of Service $(\mathrm{CoS})$, and the bandwidth of the connection. When determining the route, signaling is required to establish, teardown, maintain, and restore the connections when there is event of fault (Ellinas et al. 2011). 
There are three types of the GMPLS Signaling Protocols that are the following

Resource Reservation Protocol with Traffic Engineering extensions (RSVP-TE)

The RSVP is a signaling protocol to make use the path which computed by such routing protocol as OSPF to reserve the indispensable network resources for a session's establishment which supports both multicast traffic, and point-to-point in IP networks with requirements of the QoS (Ellinas et al. 2011; Zhang et al. 1997).

The traffic engineering TE and RSVP (RSVP-TE) is an extension that supports the management and the establishment of LSP tunnels including the stipulation of the traffic characteristics and attributes, and the stipulation of explicit route of the LSP as well as supporting the fault detection capablilities by sending a Hello message between adjacent Label Switched Routers (LSRs) (Awduche et al. 2001). The RSVP is accommodated for the independence between the date and control planes, and adapted for circuit-switched rather than packet switched connections to support a set of multiplexing and switching types such as fiber switching, waveband switching, and wavelength switching (Banerjee et al. 2001; Ellinas et al. 2011).

Signaling for Lightpath Establishment

Path and Resv are used as it is in the MPLS networks to establish lightpaths in the optical networks. The message of the Path carries extra information to be provisioned and forwarded downstream from the source to the destination node that processed at intermediate nodes. A Resv message is created after it reaches the destination node and forwarded upstream. The Resv message the cross-connects are processing at each intermediate node and set in order to establish a bi-directional connection (Ellinas et al. 2011).

Signaling for protection/restoration

Where the protection is provided in the networks and during the connection provisioning phase, the GMPLS signaling can be utilized during the connection phase to provision the secondary protection paths (Banerjee et al. 2001). When detecting any failure by the source in the case of alternative paths, the Notify message, which contains the failed connection information, is used along with GMPLS RSVP-TE to reactivate the precomputed protection path and this process called the restoration (Banerjee et al. 2001).

\section{IP OVER WDM NETWORK ARCHITECTURE}

The rapidly extraordinary growth in the Internet traffic beside the opulence of transmission range granted by WDM has an indication of the beginning of a new networking model where the imagination of combination of the optical networking and data seems to be indispensable (Ali et al. 2001; Ghani, Dixit \& Wang 2000). The IP/ WDM networking architecture makes a combination of the strengths of both optical transport and IP where the IP implements directly over the WDM in order to bypass the intermediate layer technology. Such advances as IP/MPLS routers in the WDM of data-centric equipment and optical networking technology, have increased its attention that being driven to the topic of Optical Networking on how optimal interaction can be done at these layers.

The implementation of the data directly over optics has eliminated the unnecessary of the network layers that lead to a significant reduction in the complexity and cost of the network which has led to migrate from multi-layer networking architectures to two-layer networking architecture (IP/WDM). The migration allows to interface high-performance directly to Optical Transport Network (OTN) which reduces the role of Synchronous Digital Hierarch/Synchronous Optical Network (SDH/SONET). Although, the elimination of some layers, the important functionalities which is provided by the intermediate layers such as fast restoration and multiplexing in SONET, and traffic engineering in ATM must kept to be used in the future IP/WDM networks which can be performed 
in one of the following three ways

A. By distributing the functionalities between the optical and IP layer.

B. By moving them down to the optical layer.

C. By moving them up to the IP layer

\section{An Optical Layer-Based Unified Control Plane Architecture}

Ellinas etal.2011) stated that the "ideally, the control plane functionalities should be integrated and implemented at one and only one layer". Since the thought of the network intelligence movement up to the IP layer of routers over optical switches, an optical layer-based unified control plane architecture model describes the network functionality movement and intelligence down to the optical layer of switches over routers which is more coercive in terms of overall cost savings, scalability, simplicity, and feasibility for near-term deployment. An optical layer-based control plane is utilized by this model that manage the network elements in transport layers and client while the complete separation between the layers is still retaining in the (analogous to the overlay) model. This means that the unified model retains the advantages of these two models along with avoiding their limitations (Ellinas et al. 2011; Khalil et al. 2003).

The Optical core can be classified as Autonomous System which is hidden entirely from domain, which means that both physical and logical layers belong to a monocular administrative domain while the other networking intelligence belongs to the optical layer. The unified control plane can be supported by GMPLS protocol to provide sub-lambda and full-lambda signaling, routing, and survivability functionalities(Ellinas et al. 2011). All the network resources are visible when deploying the unified control model, thus, using more efficient resources can be achieved in managing, provisioning, and restoring the connection. (Ellinas et al. 2011) mention that, adapting the unified control plane interconnection model can open up new horizons that help to implement several notable novel applications that can substantially transform and magnify the image of the next generation optical Internet such as

- The network is now capable of being managed end-to-end across multiple Autonomous Systems (Ellinas et al. 2011).

- The physical layer can restore both router and link/OXC failures at any granularity (sub lambdas and/ or full lambdas) (Ellinas et al. 2011).

- Optical Ethernet (GigE/WDM) network infrastructures where native Ethernet frames are routed directly over WDM can be developed, by consolidating the cost effectiveness and simplicity of Ethernet Technology with the ultimate intelligence of the WDM-based optical transport layer (Ellinas et al. 2011).

\section{Node architecture for the unified control plane}

The main intelligence component of the Unified Control Plane model is the OXC controller which is responsible for updating, maintaining, and creating both the logical and physical connectivity as well as the capability of provisioning on-demand lightpaths and low-speed connection requests (Ellinas et al. 2011).

\section{Optical Layer-Based Provisioning}

The Optical Transport Network in this model is responsible for providing the provisioning services in the way that it believes optimum. For example it can provide the following:

- Open up one or more than lightpaths to service the new traffic (Ellinas et al. 2011).

- Use one or more of the existing lightpaths to multiplex the new traffic on (Ellinas et al. 2011).

- Use a combination of existing lightpaths together with setting up new ones to serve the new traffic, which called a hybrid provisioning (Ellinas et al. 2011). 
Physical provisioning

Physical provisioning is used to solve the problems of the Routing and Wavelength Assignment (RWA). This is made up of two sub-problems as the name implies which deal with the assigning wavelength channels, and the path discovery (routing) along its fiber links. There are many ways are proposed to solve the RWA problems including trying to solve the problem simultaneously, or treating the sub-problems separately. When attempts to solve the RWA problems concurrently, a dynamic routing, and wavelength assignment algorithm can be utilized by means of dynamic routing over multi-layered graphs where every layer depicts a wavelength (Ellinas et al. 2011).

\section{Logical provisioning}

The logical provisioning can be obtained by establishing lightpaths as directional logical links that comprise the logical topology that performs whenever a call is attempted to be served logically. Since the changes of the topology every time a lightpath has to be set up or torn down. During the mechanisms invoke of the logical provisioning; the logical topology is checked for availability of a route spanning multiple logical links (multi-hops) or single logical link (single hop) sequentially (Ellinas et al. 2011). For the routing algorithm, the logical link cost over the logical topology can be based on the standard used of the bandwidth if the link after accommodating the call. There are some actions that can be taken after checking the logical topology, pruning and deletion from the topology will be performed for the links that do not have enough bandwidth to accommodate the call, or a node whose IP module residual speed is not sufficient to forward the call (Ellinas et al. 2011).

\section{Hybrid provisioning}

The integrated routing approach that combines resource information across the layers is utilized by the unified control model, thus, the calls can be serviced as a mixture of logical and physical routing (hybrid provisioning) in order to minimize the usage of the network resources as illustrated in the Figure 1 (Ellinas et al. 2011). There are two wavelength channels available on each link of lambda1 and lambda2, and every call is a sub-lambda request. As shown in Figure 1, assume that node A make call 1 to node E which arrives at the network first. By invoking the RWA, the lightpath is set up on wavelength lambda1 between node A and node B (Ellinas et al. 2011). When call 2 from node $B$ to node $C$ arrives into the network requesting servie, this call can only be serviced by RWA on the new lightpath on wavelength lambda2 (Ellinas et al. 2011). Assuming that call 3 from node $\mathrm{A}$ to node $\mathrm{F}$ arrives at the network and requests service, this call would have been blocked since the link between node $\mathrm{B}$ and node $\mathrm{C}$ is completely utilized at the wavelength level and there is no logical connectivity exists between node A and node F (Ellinas et al. 2011). Although, under a hybrid provisioning approach, the optical core is aware of both the physical and logical topology and can utilize the already established ligtpath between node $\mathrm{A}$ and node $\mathrm{E}$ and try to complete the remaining portion from nodes $\mathrm{E}$ to $\mathrm{F}$ on wavelength lambda2 by means of the RWA (Ellinas et al. 2011).

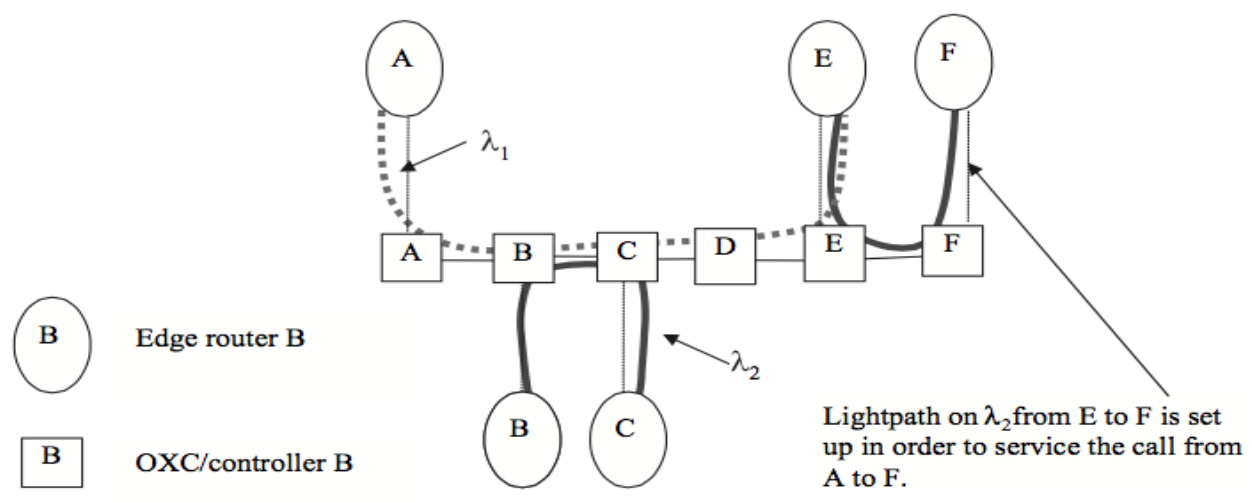

Figure 1. Concept of hybrid provisioning. Adapted from (Ellinas et al. 2011)

Volume 1

Page 5 


\section{CONCLUSION}

In conclusion of this research paper, the Generalized MPLS has been widely presented and viewed as a basis Protocols for the Optical Control Plane networks. The unified optical layer-based control for IP over WDM network architecture are also presented and discussed and specifically the Node architecture for the unified control plane, Optical Layer-Based Provisioning, Physical provisioning, Logical provisioning, and Hybrid provisioning.

\section{REFERENCES}

Ali, MA, Shami, A, Assi, C, Ye, Y \& Kurtz, R 2001, 'Architectural options for the next-generation networking paradigm: is optical Internet the answer?', Photonic Network Communications, vol. 3, no. 1-2, pp. 7-21.

Awduche, D, Berger, L, Gan, D, Li, T, Srinivasan, V \& Swallow, G 2001, RSVP-TE: extensions to RSVP for LSP tunnels.

Awduche, D \& Rekhter, Y 2001, 'Multiprotocol lambda switching: combining MPLS traffic engineering control with optical crossconnects', Communications Magazine, IEEE, vol. 39, no. 3, pp. 111-116.

Banerjee, A, Drake, J, Lang, J, Turner, B, Awduche, D, Berger, L, Kompella, K \& Rekhter, Y 2001, 'Generalized multiprotocol label switching: an overview of signaling enhancements and recovery techniques', Communications Magazine, IEEE, vol. 39, no. 7, pp. 144-151.

Dijkstra, EW 1959, 'A note on two problems in connexion with graphs', Numerische mathematik, vol. 1, no. 1, pp. 269-271.

Ellinas, G, Hadjiantonis, A, Khalil, A, Antoniades, N \& Ali, MA 2011, ' 3 The optical control plane and a novel unified control plane architecture for IP/WDM networks', Next-Generation Internet: Architectures and Protocols, p. 42.

Ghani, N, Dixit, S \& Wang, T 2000, 'On IP-over-WDM integration', Communications Magazine, IEEE, vol. 38, no. 3, pp. 72-84.

Khalil, A., Hadjiantonis, A., Ellinas, G., \& Ali, M. 2003, 'A novel IP-over-optical network interconnection model for the next-generation optical internet', Global Telecommunications Conference, 2003. GLOBECOM'03. IEEE.

Kompella, K, Katz, D \& Yeung, DM 2003, 'Traffic Engineering (TE) Extensions to OSPF Version 2', Internet Engineering Task Force (IETF) RFC 3630.

Kompella, K, Berger, L \& Rekhter, Y 2005, 'Link bundling in MPLS traffic engineering (TE)', Internet Engineering Task Force (IETF) RFC 4201.

Lang, JP 2005, 'Link management protocol (LMP)', Internet Engineer- ing Task Force (IETF) RFC 4204.

Strand, J 2005, 'Impairments and other constraints on optical layer routing', Internet Engineering Task Force (IETF) RFC 4054.

Swallow, G, Ishimatsu, H, Rekhter, Y \& Drake, J 2005, 'Generalized multiprotocol label switching (GMPLS) user-network interface (UNI): resource reservation protocol-traffic engineering (RSVP-TE) support for the overlay model', Internet Engineering Task Force (IETF) RFC 4208.

Zhang, L, Berson, S, Herzog, S \& Jamin, S 1997, 'Resource ReSerVation protocol (RSVP)', Internet Engineering Task Force (IETF) RFC 2205.

Citation: Abdullah Alsulami, "An Overview of Optical Control Plane and Novel Unified Control Plane Architecture for IP/WDM Networks". American Research Journal of Computer Science and Information Technology, Volume 1; pp: 1-6.

Copyright (c) 2016 Abdullah Alsulami, This is an open access article distributed under the Creative Commons Attribution License, which permits unrestricted use, distribution, and reproduction in any medium, provided the original work is properly cited.

Volume 1

Page 6 